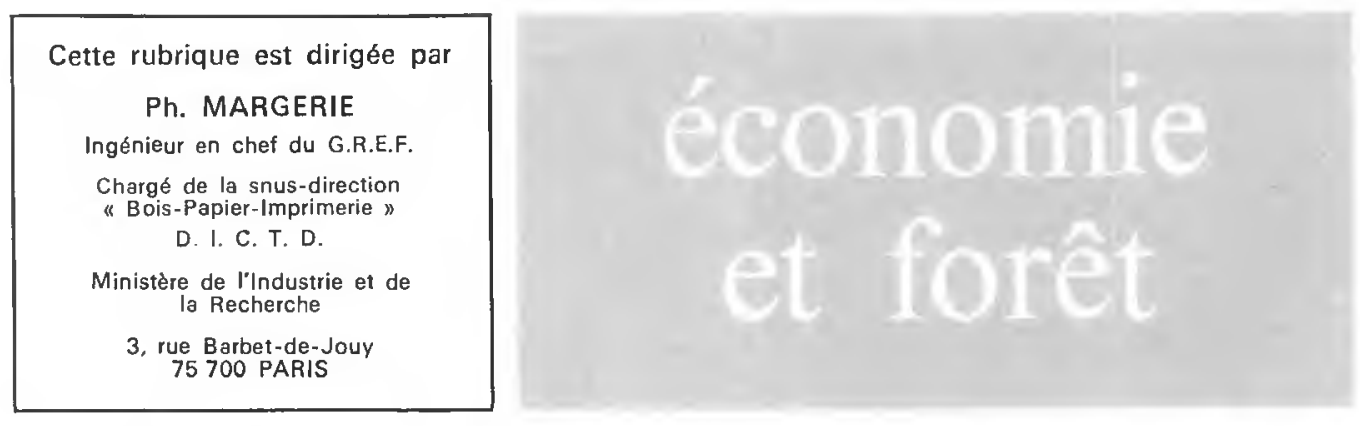

\title{
LES PAPETERIES DE GASCOGNE
}

\author{
Y. du CHEYRON d'ABZAC
}

Class. Oxford 861 (44 $\times$ Aquitaine)

N.D.L.R. - // nous a semblé intéressant de présenter de temps en temps à nos lecteurs une entreprise française. L'article qui suit, écrit pour la Revue forestière française par le responsable des relations publiques des Papeteries de Gascogne, présente l'histoire et les activités d"une importante Société industriel/e bien connue des propriétaires et des forestiers. Bien entendu, comme pour les autres articles, la Revue forestière française laisse à l'auteur la responsabilité de son texte et de ses conclusions.

\section{UNE ORIGINE RÉGIONALE}

Les Papeteries de Gascogne ont plus de cinquante ans. D'abord une petite entreprise régionale créée en 1925, afin de valoriser la forêt landaise par le biais de l'industrialisation; aujourd'hui un groupe largement diversifié dont le rayonnement s'étend non seulement à la France tout entière mais également à l'Europe.

Pourtant, en un demi-siècle d'une évolution qui l'a mené notamment au premier rang des producteurs européens de papier kraft d'emballage souple, le groupe n'a cessé d'affirmer une vocation résolument régionale : gascon il est, gascon il se veut. Un attachement à la terre d'origine qui s'exprime aussi bien dans le choix géographique des implantations que dans la répartition du capital : la très grande majorité des 640000 titres de la Société étant localisée dans le Sud-Ouest et plus particulièrement dans le département des Landes.

\section{Le défi gascon}

1925 : le bois se vend mal, les cours s'effondrent, les Landes sont au bord de la misère... Ce sont les Landais eux-mêmes qui vont relever le défi : des propriétaires forestiers soucieux de redonner vie à leur région. Leur idée : fabriquer sur place du papier kraft écru d'emballage à partir du pin maritime. Leurs moyens : une modeste usine -7000 t de papier en 1927. Et tout de suite le cortège des difficultés : financières, techniques... Mais les Papeteries de 
Gascogne sont nées, et avec elles une volonté, une ténacité qui ne se démentiront plus : affirmer au travers d'une réalisation industrielle les vertus d'une province.

$10000 \mathrm{t}$ de papier en 1945, $30700 \mathrm{t}$ en 1957... « Gascogne » a tenu son pari. A partir de cette époque, aux vertus originelles vont s'adjoindre les méthodes modernes de gestion et une stratégie de développement appuyée sur le puissant massif forestier gascon.

En effet, le premier objectif du président Louis Blanc fut de resituer en quelque sorte la société dans son environnement immédiat - la forêt landaise - par l'acquisition d'un patrimoine forestier autonome, la valorisation des « sous-produits» sciage, la transformation (Escobois, filiale de "Gascogne " fabrique près d'un million de mètres carrés de parquets et de lambris); mais aussi par une activité de recherches, d'éducation et de services. C'est ainsi que le Centre d'études des techniques forestières diffuse largement les méthodes modernes de sylviculture et le Bureau de coopération forestière se charge de la gestion des parcelles des propriétaires qui le lui demandent.

\section{0 t de papier}

Démarche logique car bois et papier sont indissolublement liés : il faut $2,8 \mathrm{t}$ de grumes pour fabriquer une tonne de papier.

Ainsi, au cours de l'exercice 1974, la fabrication de la pâte a absorbé quelque 320000 t de pins pour assurer une production de 115700 t de papier.

L'usine de Mimizan, où une sixième machine à papier a été installée en 1977, voit sa capacité de production portée aux environs de 150000 t de papier.

Les besoins en bois sont budgétés à $350000 \mathrm{t}$ pour 1977 .

C'est le kraft d'emballage qui a fait la réputation de « Gascogne ». D'une part pour $80 \%$, un kraft vergé frictionné, papier noble aux usages tels que sacs de petite $\epsilon$ t moyenne contenance, enveloppes, fardelage, support d'enduction, etc. D'autre part un kraft apprêté aux caractéristiques mécaniques supérieures, mais de moins belle apparence, destiné aux industries de transformation et, surtout, aux sacs «grande contenance ».

\section{La diversification}

La prudence est une vertu provinciale, tout comme la raison, des vertus solides aussi. Et, de fait, c'est bien ainsi qu'il faut envisager la diversification que « Gascogne » a entreprise depuis une décennie. Car si les produits se sont multipliés, c'est dans la cohérence, autour et au-delà du papier, et dans un domaine affirmé comme la vocation du Groupe : la feuille souple d'emballage. Prudence et raison qui, toutefois, n'excluent ni le dynamisme, ni l'innovation.

Déjà en 1952 "Gascogne » avait investi dans la transformation par la création, à Mimizan, d'une sacherie. Celle-ci est aujourd'hui la plus importante de France. Avec une capacité de $35000 \mathrm{t}$, elle couvre $15 \%$ du marché national. A son actif, un double titre de gloire : l'oscar de l'emballage, obtenu à deux reprises.

En 1963, "Gascogne » s'engageait plus avant dans la transformation en transférant à Dax, dans une usine moderne, les activités de la Société provençale d'aluminium (SOPAL) dont elle venait de prendre le contrôle. Un parc de machines puissantes et ultra-modernes potentiel de production 350 à 300 millions de $\mathrm{m}^{2} / \mathrm{an}$ - un laboratoire d'essais et de recherches et plus de dix années d'expérience ont fait de SOPAL une véritable entreprise de pointe dans sa spécialité, les complexes en feuille souple, les enductions de PVDC qui donnent au papier d'excellentes propriétés d'imperméabilité et d'étanchéité, les contre-collés papier aluminium, les papiers gommés, les papiers à usages industriels (papiers transferts, par exemple, utilisés dans l'industrie des cuirs synthétiques), les complexes polyéthylène extrudés (extrusion lamination). 


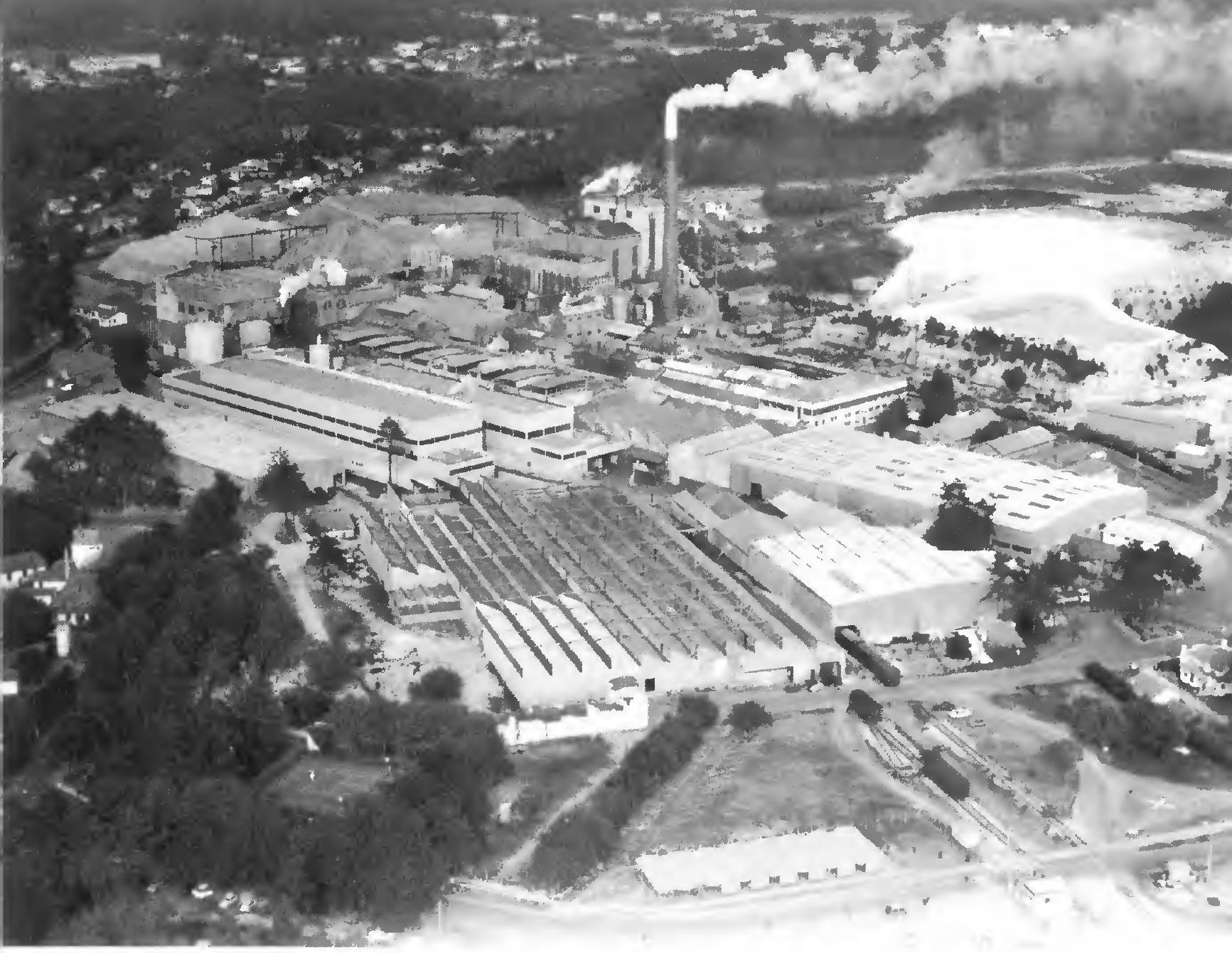

Papeteries de Gascogne. Usine de Mimizan

Photo SCHMIDT

\section{RÉPARTITION DES ACTIVITÉS}

Année 1955

Chiffre d'affaires H.T.

non consolidé

$25145681 \mathrm{~F}$

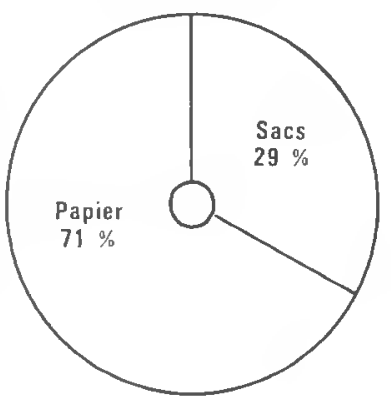

Annèe 1965

Chiffre d'affaires H.T.

non consolidé

$107617251 \mathrm{~F}$

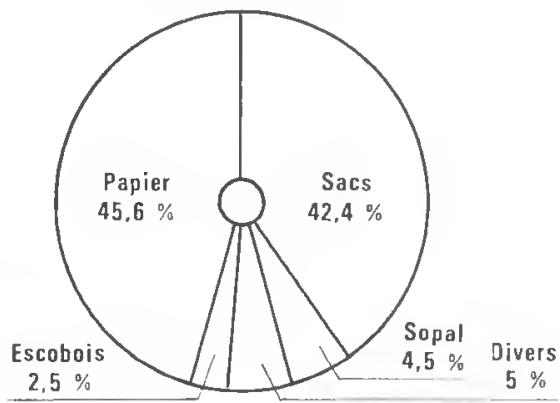

Année 1975

Chiffre d'affaires H.T

consolidé

$355558158 \mathrm{~F}$

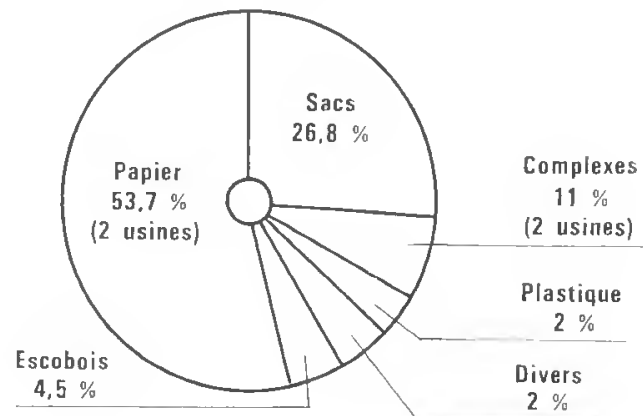


En Espagne (Logrono) SOPAL Espagne, filiale commune des Papeteries de Gascogne, de SOPAL France et de la Société espagnole Torrealba y Bezares, possède une infrastructure industrielle lui permettant une bonne pénétration sur le marché espagnol.

\section{Vers le plastique}

Peut-on, de nos jours, ignorer le plastique dans une profession telle que l'emballage? La stratégie de "Gascogne » a été guidée par une idée simple : si papier et plastique sont souvent concurrents, ils sont surtout complémentaires. Choisir le plastique, ce n'était donc pas se lancer, par une sorte d'esprit opportuniste, dans une voie étrangère mais, bien au contraire, aborder en son temps, une nouvelle étape d'une évolution harmonieuse, aller non pas "hors " du papier mais "au-delà » du papier.

Ce fut d'abord le stade expérimental matérialisé par une série de travaux effectués au Centre de recherche et d'essais de Dax, puis fut créée la Société dacquoise d'applications plastiques SODAP, dont l'usine a été achevée au cours de l'été 1976 et dont la capacité initiale de 8000 à 10000 t par an est susceptible d'accroissement suivant les besoins du marché.

Des produits donc, des performances même puisqu'au cours de ces dernières années, en dépit des difficultés conjoncturelles que connaissait la profession, "Gascogne " n’a pas cessé pour autant ses investissements, affirmant ainsi sa foi en l'avenir; des projets aussi, dont la SODAP est une des manifestations les plus évidentes...

\section{La part du bois}

L'ensemble des activités du groupe étant sommairement indiqué, il convient, pour les lecteurs de la Revue forestière française, d'examiner un peu plus en détail le secteur bois et forêt.

II comprend essentiellement :

- une des trois divisions de la société mère des Papeteries de Gascogne:

— deux filiales de cette société : la Société forestière de Gascogne et Escobois.

\section{La division bois des papeteries de Gascogne}

La société des Papeteries de Gascogne est fractionnée en trois divisions autonomes : boispapier-sacs.

Placé sous l'autorité de M. Claude Lefebvre, directeur, la division «Bois " a pour mission d'assurer, aux meilleures conditions de qualité et de prix, l'approvisionnement en matières premières de la papeterie de Mimizan (division "Papier») et des autres sociétés du groupe qui seraient demandeurs de bois.

Son organisation interne propre lui permet de faire face à ses différentes activités qui sont principalement :

- les achats de bois. Dans le massif gascon pour 60 à $65 \%$ des besoins, dans l'ouest de la France et dans le Massif Central ou éventuellement à l'étranger pour le surplus. Ces achats peuvent être directs ou par l'intermédiaire du négoce; ils se font soit sur pied soit bord de route, soit auprès des industries pour les croûtes, délignures et autres déchets ;

- l'exploitation forestière. Planification des coupes-vidange des chantiers et transport du bois après répartition des billons entre sciage et trituration.

- la coopération forestière, en liaison avec les organismes régionaux (Institut du pin) ou nationaux au profit des sylviculteurs locaux (Centre d'étude des techniques forestières des Landes) et la gestion de propriété à la demande et pour le compte des propriétaires qui ne peuvent le faire eux-mêmes;

- la gestion de ses personnels. La division "Bois" emploie environ 200 salariés dont 16 cadres et ingénieurs et 22 techniciens et employés.

- la gestion financière. La division "Bois " a un des plus gros postes budgétaires de la société avec un total supérieur à 10 milliards d'anciens francs. 


\section{Les filiales bois du groupe Gascogne}

Elles sont au nombre de deux.

- La plus ancienne est la Société “Escobois» qui, dans son usine d'Escource (Landes) fabrique principalement des parquets et lambris en pin des Landes. La société Escobois coiffe également les activités de sciage du groupe «Gascogne ».

En 1976 Escobois a produit $600000 \mathrm{~m}^{2}$ de lambris et $225000 \mathrm{~m}^{2}$ de parquet. Son chiffre d'affaires a été supérieur à $24000000 \mathrm{~F}$. L'usine d'Escource a continué sa modernisation par l'installation d'une deuxième chaîne de fabrication de lambris. Elle est également équipée de deux séchoirs à bois, par fluide thermique, d'une capacité totale de $160 \mathrm{~m}^{3}$. Escobois commercialise $60 \%$ de sa production par ventes directes et $40 \%$ par l'intermédiaire de commissionnaires. $11 \%$ de sa production est vendu à l'exportation principalement en Belgique, Espagne et République fédérale allemande. 1976 a vu également le lancement d'un panneau décoratif " ESCO $3 \mathrm{~L}$ » qui est fort bien accueilli par la clientèle.

L'activité de sciage se fait sur huit métiers en quatre points du massif landais à Ychoux, Escource, Moliets et Uza. Avec une production de $30500 \mathrm{~m}^{3}$, Escobois est un des plus importants scieurs des Landes.

Escobois emploie 52 personnes, 25 autres sont employées par les scieries.

- La deuxième filiale est la "Société forestière de Gascogne " dont la création ne remonte qu'au mois de décembre 1976. Cette société anonyme à responsabilité limitée au capital de $12000000 \mathrm{~F}$ a été constituée par apport de la totalité du patrimoine forestier du groupe (2 500 ha).

\section{UNE VOCATION EUROPÉENNE}

\section{Une fédération}

Toutefois, on remarquera qu'à tous les niveaux, malgré un développement exemplaire, les Papeteries de Gascogne ont su échapper au gigantisme. Qu'il s'agisse de la papeterie proprement dite, qu'il s'agisse de la division sacs, de SOPAL ou encore d'ESCOBOIS, le groupe se veut comme un ensemble fédéré de "cellules à taille humaine ». Une entreprise à l'échelle de I'homme, un outil au service d'une région.

Fédérer, c'est d'ailleurs le grand principe qui semble avoir guidé toute l'action commerciale de "Gascogne ", à tel titre qu'il faudrait plutôt parler de philosophie que de politique commerciale.

«Dans le concurrent, déclare-t-on aux Papeteries de Gascogne, nous ne voulons pas plus voir un ennemi que nous ne voulons voir une proie dans la petite entreprise cliente. Dans l'un et l'autre, nous voyons un partenaire, c'est-à-dire une partie prenante de l'organisation du marché. »

D'où le respect de "Gascogne » pour ses clients quels qu'ils soient et quelle que soit leur importance et son souci de créer des rolations non seulement avec eux mais entre eux - de fédérer un ensemble qui ne demandait qu'à dovenir homogène. Depuis 1968, le Groupement d'intérêt économique de l'emballage (GEMBAL) remplit ce rôle : 29 distributeurs et transformateurs réunis au sein d'un organisme qui permet de coordonner les actions respectives et laisse à chacun toute son indépendance. GEMBAL distribue notamment plus de la moitié du papier d'emballage qui est fabriqué en France.

Une efficacité qui d'ailleurs ne s'arrête pas là. Avec GEMBAL, le groupe a trouvé le cadre où mettre en œuvre l'autre aspect de sa politique : vendre au-delà des produits, des services ; services en quelque sorte directs lorsqu'il s'agit du façonnage très poussé du papier effectué 
à Mimizan ou des stocks mis en dépôt chez certains clients; mais services beaucoup plus importants encore quand il s'agit de faire en commun des études de marketing, d'harmoniser les méthodes et systèmes de gestion, d'améliorer les prix de revient, d'assurer les ventes, etc.

\section{Des Landes à I'Europe}

En vue d'accroître l'efficacité de l'ensemble des dispositifs de la force de vente des productions de kraft, et ce, à l'échelle européenne (six délégations en France et sept dans le reste de I'Europe) un G.I.E. Eurolandes a été créé en 1973 par les Papeteries de Gascogne et la Cellulose du pin.

L'activité d'Eurolandes tournée vers le marché européen et permettant une action continue, homogène, au niveau de l'ensemble de la clientèle, utilisant ou susceptible d'utiliser les papiers kraft, a donné une nouvelle dimension à l'activité de base.

La conception même et les méthodes de travail d'Eurolandes permettent d'associer l'efficacité indispensable à la commercialisation de produits industriels de masse (papiers kraft) à la nécessité du maintien d'une approche à taille humaine de l'ensemble des problèmes commerciaux.

Et l'avenir? D'abord continuer, se maintenir à cette place originale qu'ont valu à "Gascogne \# ses choix et ses efforts. Nouveau président depuis l'année 1975, M. Philippe Blanc a la même volonté affirmée que l'entreprise demeure humaine, tant par la taille que par l'esprit. En vérité, ce que "Gascogne » recherche, ce n'est pas la croissance pour la croissance, souvent anarchique, mais une sorte d'évolution réfléchie de tous les éléments qui la composent. Elle l'a prouvé et entend bien le prouver encore. C'est à peu près ce qui ressort de ce que l'on pourrait appeler le schéma de développement des prochaines années dont les principaux points sont les suivants :

- $150000 \mathrm{t}$ de papier d'emballage à Mimizan :

- la consolidation et le développement possibles, suivant la conjoncture, de SODAP :

- I'achèvement du plan de développement 1973-1978 de SOPAL qui doublera sa capacité de production.

En outre, un accord a été conclu entre SOPAL et les papeteries René Sibille qui ont apporté à SOPAL leur activité de transformation spécialisée dans les technologies complémentaires à celles pratiquées par la SOPAL.

L'âge de la puissance? de la sagesse? Certainement, mais déjà toute l'histoire des Papeteries de Gascogne se réclame d'elles. L'âge de la solidité aussi, la résistance du groupe à la dernière crise, et sa rapidité à en tirer les conséquences l'ont bien montré...

La solidité d'une terre, d'une forêt, la plus vaste de l'Europe des Neuf où « Gascogne » est née. La solidité d'un actionnariat régional qui soutient puissamment le groupe.

"Gascogne » est probablement la seule entreprise française dont l'assemblée générale réunit chaque année plus de cinq cents personnes, auxquelles est offerte un repas digne de la région. C'est à partir de cet environnement que le groupe organise son avenir et rayonne brillamment sur les marchés européens.

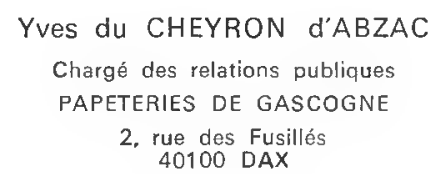

